\title{
A method for studying shape change in children
}

\author{
HARVEY GOLDSTEIN \\ University of London Institute of Education \\ and FRANCIS E. JOHNSTON \\ Department of Anthropology, University of Pennsylvania
}

[Received 5 May 1977; revised 23 August 1977]

Summary. A method is given for measuring shape change with age, utilizing two dimensional body outlines. Problems of standardization for overall size are discussed and it is shown how the method can be used to draw D'Arcy Thompson-type transformation grids. The method is applied to serial measurements on a girl from $3 \frac{1}{2}$ to 19 years of age.

\section{Introduction}

One of the first serious attempts to study the shapes of biological organisms was made by D'Arcy Thompson (1917). He considered two-dimensional diagrammatic representations and studied ways in which diagrams based on different organisms were related-his well known "transformation grids". Here shape is defined in terms of the geometrical relationships between corresponding features of two diagrams. An alternative development, mainly developed by Huxley (1932), and known as allometry, defines "shape" in terms of relationships among a set of lengths of body segments. Huxley's ideas were often applied to relationships observed in growing individuals, whereas Thompson's ideas found most application in the comparison of different species.

The original ideas of "bivariate" allometry, where only two lengths were related have undergone considerable mathematical development, have been generalized to the case of several lengths, and found applications in several areas (see, e.g., Sprent, 1972). Thompson's ideas on the other hand, seem not to have aroused so much interest, and it is the purpose of this paper to apply a development of his ideas to the study of shape change in children. This development is originally due to the work of Sneath (1967), and in the next section his methods are summarized.

\section{Materials and methods}

The method will be described with reference to the shapes of children using frontal pictures in a standard pose (see Tanner, 1955, pp. 161-164, for details). Figure 1 shows the shape outline of a $3 \frac{1}{2}$ year old girl copied from a photo. In successive outlines it is possible to identify corresponding points on the perimeter of the outline, such as the vertical surface of the neck, the most lateral extension of the shoulder, and so on. Changes in shape will be reflected in these changing distances.

Before we can properly study shape, however, some account must be taken of differences in overall size. If, for example, all the linear dimensions in figure 1 were doubled, this would not change the 'shape' of the figure, merely the size. If, on the 
other hand, the distances in the horizontal direction only were doubled then we would perceive this a change in shape as well as size.

Several approaches to size standardization are possible. One is to join the points by straight lines and multiply all the dimensions by a constant, such that the resulting shape encloses a specified standard area. Another method is to consider the distances of the points from the centroid of the diagram and to reduce the dimensions by the same factor until the sum of the squared distances achieves a specific value. When two diagrams have the same shape, then these methods are of course equivalent.

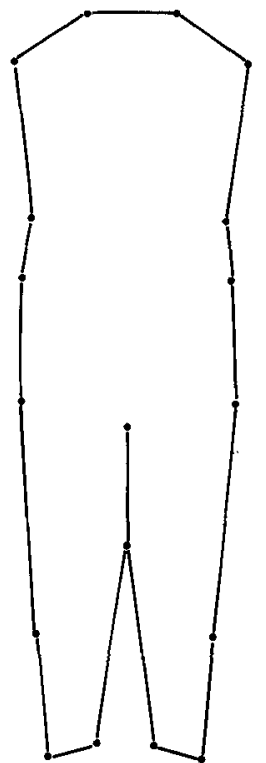

Figure 1. Shape outline of a $3 \frac{1}{2}$ year-old girl taken from a standardized photograph.

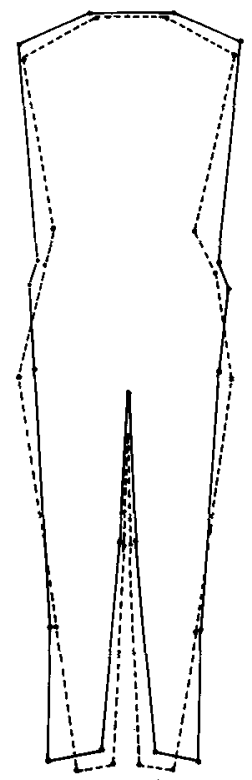

Figure 2. Shape diagrams of a girl aged $3 \cdot 5$ years (continuous) and $19 \cdot 0$ years (dashed) after standardizing for size and orientation. Superimposition is based upon the respective centroids.

Sneath adopts the latter method and we follow his example, especially since computationally the method is straightforward and involves only the calculation of the sum of squared distances from the centroid of the diagram, and then the division of each distance by the square root of this quantity. If any two diagrams have the same shape they can, after size standardization, be exactly superimposed after first orientating them to "match up". Where the shapes differ, it is necessary to carry out a matching-up to ensure as nearly as possible the same orientation, before comparing shapes. Sneath gives details of how this can be done mathematically. His method involves finding the relative orientations so that the sum of squared distances between corresponding points is as small as possible. This sum of squares can be used as a measure of the shape difference between the diagrams. We denote this measure of shape difference between diagrams 1 and 2 as $D^{2}{ }_{1,2}$. For a growing child, the quantity $D_{t_{1}, t_{2}}$ is a measure of shape change between ages $t_{1}$ and $t_{2}$ and we may define a shape "velocity" as the quantity $D_{t_{1}, t_{2}} /\left(t_{2}-t_{1}\right)$.

Also the shape at each age can be referred to the adult shape as a standard, 
enabling us to measure how far from adult shape a child is. We denote this at age $H$ by $D_{H}$.

In the next section we illustrate these procedures using longitudinal data. In addition to obtaining an overall measure of shape difference we can study the manner in which the positions of the points on one diagram are related to those on another. Figure 2 shows the diagram for the 3.5 year old of figure 1 superimposed on that of the same girl at age 19 after size standardization.

If we consider the horizontal $(x)$ and vertical $(y)$ directions separately, we can relate say the $x$ values of the 19 year old diagram to the $x$ and $y$ values of the $3 \cdot 5$ year diagram, hopefully finding a simple relationship, e.g. a linear one, which will summarize the detailed shape change. If, instead of applying this transformation to the points we apply it to a rectangular coordinate grid placed over the 3.5 years diagram, we have a transformed coordinate grid at 19 years. This is a D'Arcy Thompson-type transformation grid and the present method can be viewed as a convenient way of constructing it.

\section{Results}

Consecutive shape and size changes have been calculated on children from the Harpenden Growth Study (Tanner et al., 1976). Because of the considerable labour involved in analysing all the photographs, only a few children have been studied so far. It is hoped eventually that a large representative sample will be analysed in order to provide standards. For now we limit ourselves to presenting and discussing the results of analysing the measurements made on one girl from 3.5 to 19.0 years, in order to illustrate the method.



Figure 3. Comparison of shape (solid) and size (dashed) at each age to adult values. Size comparisons are the percentages of adult size, $\left(S_{t} / S_{\mathrm{A}}\right) \times 100$. Shape comparisons are the values $D_{t, \mathrm{~A}}$, i.e. $D_{H}$.

Figure 3 shows the size and shape at each age compared to adult size and shape. As already indicated, size is measured by the square root of the sum of the variances in the $x$ and $y$ directions at age $t\left(S_{t}\right)$ and the comparison to the adult is accomplished by calculating the percentage size at any age relative to the adult $\left(S_{t} / S_{\mathrm{A}}\right) \times 100$. Shape 
is derived as above. The plot of size shows the adolescent spurt typical of growth measurements which are related to size, such as height and weight, with the maximum size velocity occurring at about the time of maximum weight and height velocity, which in this girl, were the same at about 13.5 years. The plot of shape shows a fairly rapidly increasing resemblance to adult shape from 3.5 to about 8 years, followed by a fairly constant level to about 17 years and a rapid increase thereafter. At about 14 years, corresponding to the maximum size velocity there appears to be a decrease in similarity to adult shape.

Unlike size, which is measured separately for each diagram, shape is only defined in terms of change between diagrams. Hence we cannot use figure 3 directly to estimate the shape changes from occasion to occasion. These are shown in figure 4.

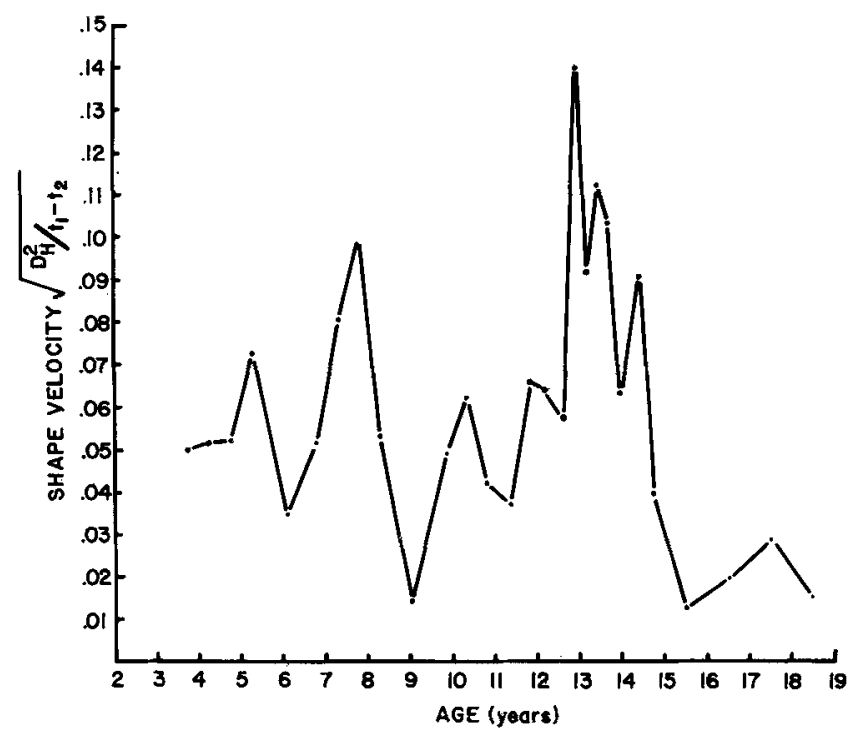

Figure 4. Shape changes between consecutive ages. Velocity of shape change from age $t_{t}$ to $t_{j}$ is given by $D_{t_{i}, t_{j}} /\left(t_{j}-t_{l}\right.$.)

The maximum velocity, as with size, is at 13-14 years with another possible peak at about 7-8 years. The spiky nature of the graph is to a large extent due to the use of all the measurements available. For the purpose of constructing standards, 1-year shape velocities would presumably be used, leading to a smoother appearance.

Polynomials were fitted relating the 19-year $x$ and $y\left(x_{19}, y_{19}\right)$ values to the $3 \cdot 5$ year $x$ and $y$ values and the following equations provided reasonable fits.

$$
\begin{gathered}
x_{19}=0.78 x-0.38 x y-0.18 x y^{2}+2.76 x^{3}+0.23 x y^{3}: \\
\text { residual mean square }=0.0004 \\
y_{19}=0.042+1.12 y-0.02 y^{2}-0.06 y^{3}-0.23 x^{2} y^{2}: \\
\text { residual mean square }=0.0016
\end{gathered}
$$

Fitting the higher order polynomials actually improved the fits somewhat (as measured by the residual mean square), and indeed we could have fitted the data exactly by including enough terms. Beyond a certain point, however, the improvement in fit is simply a question of graduating what is essentially random variation, 
including measurement errors. It is difficult to formulate criteria to determine the number of terms to fit, but the resulting equations should be as simple as possible, consistent with conveying the essential details of the shape change. We have investigated the effect of using equations with fewer terms and the results are displayed in figure 5. With the addition of higher-order terms the predicted 19-year shape approaches the observed shape. The linear equations perform rather poorly in terms of graduating the 19 year-old shape, and equations (1) perform much better.


Figure 5. Predicted (solid) and actual (dashed) shapes, and the residual mean squares, using predictive equations of increasingly higher order for relationships between $3 \frac{1}{2}$ and 19 years, as follows: (A) equations (1) of text; (B) $x_{19}=0.99 x-0.09 x y^{2}$ and $y_{19}=0.049+1.02 y-$ $0.05 y^{2}$; (C) $x_{19}=0.91 x$ and $y_{19}=0.01+1.01 y$. The numbers below the diagrams give the residual mean squares in the $x$ and $y$ directions.

Figure 6 gives the result of applying the various equations to the rectangular grid surrounding the 3.5 year-old diagram to give the 19 year-old diagrams in the form of D'Arcy Thompson-type transformation grids. These grids are shown without the usual superimposition of the shape outlines in order to present clearly the general, smoothed, nature of the shape change.

\section{Discussion}

The measure of shape we have been considering is defined only in terms of the overall divergence from congruence of two diagrams. It does not attempt to measure any particular component of shape, such as is done by the somatotyping system (Sheldon et al., 1954). Thus, for example, the difference in shape between the adult diagram and that at age 17.0 years has the same value as that between the adult diagram and the one at age $15 \cdot 0$ years. However, the diagrams at 15 years and 17 
years are not the same shape. Consecutive pairwise comparisons of shape can, however, provide further details of the rate of shape change, and the increase in this rate at about the time of maximum weight velocity suggests a possible common etiology.

The estimation of the detailed relationships between diagrams at different ages can in principle provide information about the components of shape change. The major problem here is the interpretation of what may be complicated equations. If it were possible to find simple equations relating two diagrams where say only one

A

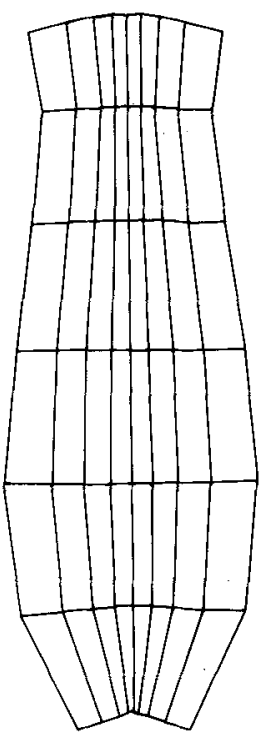

$\mathrm{B}$



C

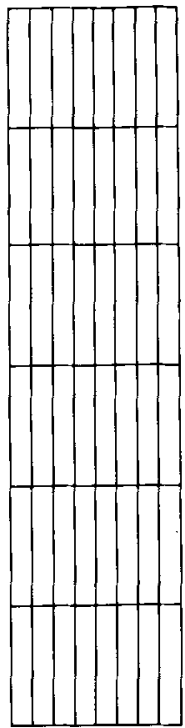

Figure 6. Transformation grids corresponding to the shapes in figure 5.

of the parameters changed from comparison to comparison, then the values of these parameters of $x$ and $y$ could be used to measure shape components. As it is, in the present case, the use of polynomials does not appear to achieve this simplicity. Moreover, even equations (1) which give a small residual error overall, do not produce an accurate shape prediction, as can be seen by noting the divergence between actual and predicted points in the hip and waist regions of figure 5. For the purpose of providing a smooth graduation between diagrams, polynomials can be used to construct D'Arcy Thompson-type transformation grids. Although therefore, the process of drawing such grids can be done routinely, there remains the problem of deciding the degree of polynomial to use. A reasonable procedure would be to continue to add higher order terms until the average residual difference between measured and predicted values reached a value which was about equal to the estimated error of measurement associated with the original diagrams.

\section{Acknowledgments}

Our thanks are due to Linda Adair for the considerable time and effort she spent preparing all the illustrations, and to Professor J. M. Tanner for his help and encouragement. 


\section{References}

Huxley, J. S. (1932). Problems of Relative Growth. London: Methuen.

Sheldon, W. H., Dupertuis, C. W., and McDermott, E. (1954). Atlas of Men. New York and London: Harper Bros.

Sneath, P. H. A. (1976). Trend-surface analysis of transformation grids. J. Zool. (London), 151, $65-122$.

Sprent, P. (1972). The mathematics of size and shape. Biometrics, 28, 23-37.

Tanner, J. M. (1955). Growth at Adolescence. Oxford: Blackwell Scientific Publications.

Tanner, J. M., Whitehouse, R. H., Marubini, E., and Resele, L. F. (1976). The adolescent growth spurt of boys and girls of the Harpenden Growth Study. Annals of Human Biology, 3, 109-126.

Thompson, D'Arcy (1917). On Growth and Form. Cambridge University Press.

Address correspondence to: Professor H. Goldstein, Department of Statistics and Computing, Institute of Education, 20 Bedford Way, London WC1H OAL.

Zusammenfassung. Es wird eine Methode zur Messung des Alterswandels der Form gegeben, wobei zweidimensionale Körperumrisse benutzt werden. Die Probleme der Standardisierung im Hinblick auf eine Gesamtgröße werden diskutiert und es wird gezeigt, wie die Methode benutzt werden kann, um Transformationsnetze in der Art von D'Arcy-Thompson zu zeichnen. Die Methode wird angewandt auf die Reihenmessung eines Mädchens von 3,5 bis 19 Jahren.

Résumé. Une méthode est donnée pour mesurer le changement de la forme avec l'âge, sur la base de profils du corps bi-dimensionnels. Les problèmes de standardisation en fonction du format général sont discutés et il est montré comment la méthode peut être employée pour dessiner des grilles de transformation du type D'arcy Thompson. La méthode est appliquée à des mensurations successives d'une fille depuis l'âge de $3 \frac{1}{2}$ ans jusqu'à 19 ans. 